\title{
Squeezing resulting from a fourth-order interaction in a degenerate parametric amplifier with absorption losses
}

\author{
P. García Fernández \\ Instituto de Estructura de la Materia, Consejo Superior de Investigaciones Científicas, \\ Serrano 123, 28006 Madrid, Spain \\ P. Colet, R. Toral, and M. San Miguel \\ Departamento Física Teórica, Universidad de Baleares, Ctra. Valldemosa, \\ 07071 Palma de Mallorca, Spain \\ F. J. Bermejo \\ Instituto de Estructura de la Materia, Consejo Superior de Investigaciones Científicas, \\ Serrano 123, 28006 Madrid, Spain \\ (Received 7 November 1990)
}

\begin{abstract}
The squeezing properties of a model of a degenerate parametric amplifier with absorption losses and an added fourth-order nonlinearity have been analyzed. The approach used consists of obtaining the Langevin equation for the optical field from the Heisenberg equation provided that a linearization procedure is valid. The steady states of the deterministic equations have been obtained and their local stability has been analyzed. The stationary covariance matrix has been calculated below and above threshold. Below threshold, a squeezed vacuum state is obtained and the nonlinear effects in the fluctuations have been taken into account by a Gaussian decoupling. In the case above threshold, a phase-squeezed coherent state is obtained and numerical simulations allowed to compute the time interval, depending on the loss parameter, on which the system jumps from one stable state to the other. Finally, the variances numerically determined have been compared with those obtained from the linearized theory and the limits of validity of the linear theory have been analyzed. It has become clear that the nonlinear contribution may perhaps be profitably used for the construction of above-threshold squeezing devices.
\end{abstract}

\section{INTRODUCTION}

The possibility of producing strong, amplitudesqueezed light by means of fourth-order interaction processes such as those responsible for the optical Kerr effect has been recently analyzed by Tanas and Kielich. ${ }^{1}$ These authors have pointed out that the squeezing effect caused by these higher-order nonlinearities can be remarkably high (up to $98 \%$ in noise reduction) provided that the incident light is sufficiently high. On the other hand, a model of a two-photon system with an added fourthorder nonlinearity has been studied ${ }^{2}$ and it was shown that it was able to produce enhanced squeezing with respect to the usual parametric devices. The model referred above can be useful to model traveling-wave devices due to the shorter times required to produce strong noise supression. The purpose of the present paper is to explore the squeezing properties of a model of a degenerate parametric amplifier (the two-photon system) where absorption losses due to the coupling at the "phonon bath" of the nonlinear material are explicitly taken into account, and where a fourth-order interaction also takes place.

The outline of the paper is as follows: the derivation of the Langevin equations is briefly described in Sec. II and a stability analysis of the deterministic limit is given in
Sec. III. The fluctuations around the steady states are analyzed in Sec. IV and the main conclusions are given in Sec. V.

The model that we have considered consists in an intense laser beam at frequency $2 \Omega$ - the pump beamthat illuminates a suitable nonlinear medium. The pump beam is assumed to be intense enough so that the undepleted pump approximation holds. The analysis was performed with the following effective $\operatorname{Hamiltonian}^{2}(\hbar=1)$ :

$$
H=\Omega a^{\dagger} a+\frac{1}{2} f(t)\left(a^{\dagger}\right)^{2}+\frac{1}{2} f^{*}(t) a^{2}+\frac{\Gamma}{2}\left(a^{\dagger}\right)^{2} a^{2}+H_{L},
$$

where $f(t)=i \kappa(t) e^{2 i(\varphi-\Omega t)} . \kappa(t)$ is an arbitrary real function of time and the second-order process is characterized by the function $\kappa(t) e^{2 i(\varphi-\Omega t)}$. The fourth-order term is known to describe optical bistability, and the coupling $\Gamma$ is proportional to the third-order susceptibility tensor of the nonlinear medium. This Hamiltonian could describe an optically bistable two-photon medium.

Absorption losses in the nonlinear material are included by means of the usual terms in the Hamiltonian ${ }^{3}$

$$
H_{L}=\sum_{j} \omega_{j} b_{j}^{\dagger} b_{j}+\sum_{j} \chi_{j}\left(b_{j} a^{\dagger}+b_{j}^{\dagger} a\right) .
$$

The Heisenberg equations for $a$ and $b_{j}$ lead to an elimina- 
tion of the heat-bath modes using the Wigner-Weisskopf approximation.

\section{LANGEVIN FORMULATION}

Although some attempts have been made in order to deal with $k$-photon anharmonic oscillator models, ${ }^{4}$ the derivation of closed-form expressions for the computation of field statistics is still an open problem. The approach taken in this paper uses a recent suggestion ${ }^{5}$ that enables us to obtain the Langevin equations for the optical fields from the Heisenberg equations provided that a linearization is introduced on heuristic grounds.

The Heisenberg equation for the annihilation operator can be linearized if the fluctuation is small in comparison with the average value. In this case we can separate in the annihilation operator the deterministic ( $c$-number) part $\bar{a}$ from the part $\delta a$, carrying the quantum fluctuations, in the form

$$
a=(\bar{a}+\delta a) e^{-2 i(\Omega t-\varphi)} .
$$

The neglect of quadratic terms in $\delta a$ transforms the nonlinear Heisenberg equation into a linear one. Then we may replace the Heisenberg operator $\delta a$ by the stochastic process $\delta \alpha$ to obtain the Langevin equation

$$
\begin{aligned}
\delta \dot{\alpha}= & -i(\Omega-i \gamma) \delta \alpha-i f(t) \delta \alpha^{*} \\
& -i \Gamma \bar{\alpha}\left(2 \bar{\alpha}^{*} \delta \alpha+\bar{\alpha} \delta \alpha^{*}\right)+L(t),
\end{aligned}
$$

where $\gamma$ is the damping constant and $L(t)$ is a complex white noise with mean and correlations

$$
\begin{aligned}
& \langle L(t)\rangle=\left\langle L(t) L\left(t^{\prime}\right)\right\rangle=0, \\
& \left\langle L(t) L^{*}\left(t^{\prime}\right)\right\rangle=2 D \delta\left(t-t^{\prime}\right) .
\end{aligned}
$$

The diffusion constant $D$ is essentially the spectral density at frequency $\Omega$ of the heat bath

$$
2 D=\gamma(1+2 \bar{n}),
$$

and $\bar{n}$ depends on the temperature of the reservoir. On the other hand, the linearization procedure will only be valid if the deterministic part $\bar{\alpha}$ verifies

$$
\dot{\bar{\alpha}}=-i(\Omega-i \gamma) \bar{\alpha}^{*}-i f(t)-i \Gamma \bar{\alpha}^{*} \bar{\alpha}^{2} .
$$

The solution of Eq. (2.2) is a Wigner probability density with an associated Fokker-Planck equation which is the same as obtained by the usual procedure starting from the quantum evolution equation of the density matrix transformed to the $W$ representation. Changing from the complex stochastic process $\alpha(t)$ to the real processes $x(t)$ and $y(t)$ through

$$
\alpha(t)=[x(t)+i y(t)] e^{-2 i(\Omega t-\varphi)},
$$

the Langevin equation before linearization becomes

$$
\begin{aligned}
& \dot{x}=(-\gamma+\kappa) x+\Gamma y\left(y^{2}+x^{2}\right)+L_{x}, \\
& \dot{y}=(-\gamma-\kappa) y-\Gamma x\left(y^{2}+x^{2}\right)+L_{y},
\end{aligned}
$$

where $L_{x, y}(t)$ are real Gaussian white noises of zero mean and correlations

$$
\left\langle L_{i}(t) L_{j}\left(t^{\prime}\right)\right\rangle=D \delta_{i j} \delta\left(t-t^{\prime}\right), \quad i, j=x, y .
$$

We take the semiclassical model (2.7) as the basis of our subsequent analysis.

\section{DETERMINISTIC EQUATIONS AND LINEAR STABILITY ANALYSIS}

By introducing the dimensionless time $\tau=t(\gamma+\kappa)$, Eqs. (2.7) adopt the simpler form:

$$
\begin{aligned}
& \frac{d x}{d \tau}=\mu x+v y\left(x^{2}+y^{2}\right)+\widetilde{L}_{x}, \\
& \frac{d y}{d \tau}=-y-v x\left(x^{2}+y^{2}\right)+\widetilde{L}_{y},
\end{aligned}
$$

where we have introduced the dimensionless constants

$$
\mu=\frac{\kappa-\gamma}{\kappa+\gamma}, \quad \nu=\frac{\Gamma}{\gamma+\kappa} .
$$

As the gain $\kappa$ and loss $\gamma$ parameters are positive definite, $\mu$ is confined to values in the interval $[-1,1]$. $\widetilde{L}_{x}$ and $\widetilde{L}_{y}$ are real Gaussian noises with zero mean and correlations given by

$$
\left\langle\widetilde{L}_{i}(\tau) \widetilde{L}_{j}\left(\tau^{\prime}\right)\right\rangle=\bar{D}_{i j} \delta\left(\tau-\tau^{\prime}\right),
$$

where the coefficients of the (diagonal) diffusion matrix $\bar{D}$ are given by $\bar{D}_{i j}=\bar{d} \delta_{i j} ; \bar{d} \equiv D /(\gamma+\kappa)$. We now find the steady states of the deterministic version of Eqs. (3.1) above. Straightforward algebra shows that these stationary solutions verify

$$
\frac{x_{s}^{2}}{y_{s}^{2}}=\frac{1}{\mu} \text {. }
$$

The system has a switch-on threshold for $\mu=0$ : For $\mu<0$ the zero intensity solution $\left(x_{s}, y_{s}\right)=(0,0)$ is the only one allowed. For $\mu>0$, in addition to this trivial solution, there also exists two nonzero intensity stationary solutions given by

$$
x_{s}= \pm \frac{\mu^{1 / 4}}{\sqrt{v(1+\mu)}}, \quad y_{s}=-\sqrt{\mu} x_{s} .
$$

Using standard techniques, the local stability of the stationary solutions is analyzed by considering small variations around them, i.e., $x=x_{s}+\delta x, y=y_{s}+\delta y$. From Eq. (3.1) (without the noise term) we finally obtain

$$
\left[\begin{array}{l}
\delta \dot{x} \\
\delta \dot{y}
\end{array}\right]=A\left[\begin{array}{l}
\delta x \\
\delta y
\end{array}\right]
$$

where the drift matrix $A$ is given by

$$
A=\left[\begin{array}{cc}
\mu+2 v y_{s} x_{s} & v\left(x_{s}^{2}+3 y_{s}^{2}\right) \\
-v\left(3 x_{s}^{2}+y_{s}^{2}\right) & -1-2 v x_{s} y_{s}
\end{array}\right] .
$$

Stability is governed by the eigenvalues of the matrix $A$, which are

$$
\begin{array}{r}
\lambda_{1,2}=\frac{1}{2}\left\{\mu-1 \pm\left[(\mu+1)^{2}+8(\mu+1) v x_{s} y_{s}\right.\right. \\
\left.\left.-12 v^{2}\left(x_{s}^{2}+y_{s}^{2}\right)^{2}\right]^{1 / 2}\right\} .
\end{array}
$$

In the case of the zero-intensity solution $\left(x_{s}, y_{s}\right)=(0,0)$ 


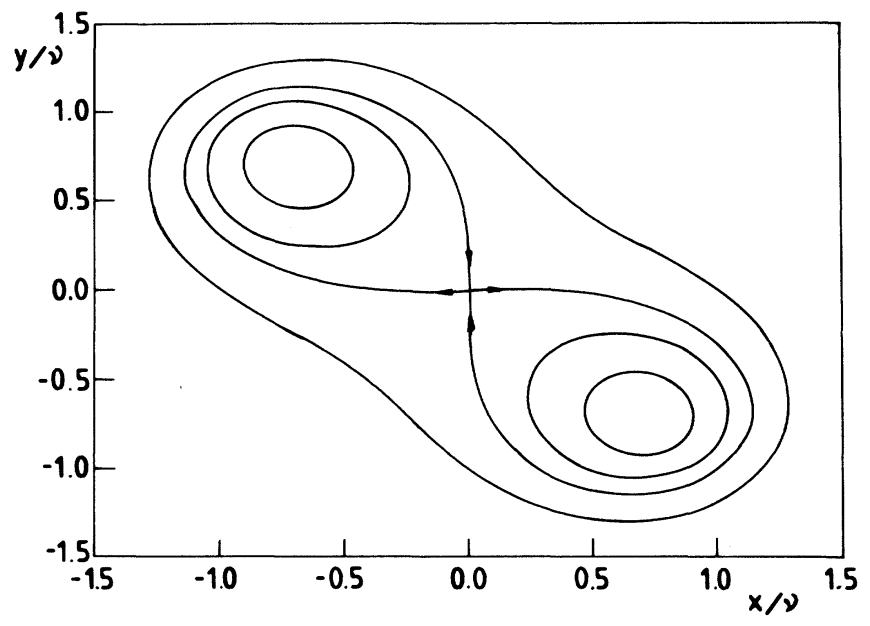

FIG. 1. Phase-space orbits of constant energy given by the Hamiltonian (3.1). From the smallest orbit to the largest one, the corresponding energies are $-0.8,-0.4,0,1.0$.

we have $\lambda_{1}=1$ and $\lambda_{2}=\mu$, such that this solution is stable below threshold $(\mu<0)$. Above threshold the zerointensity solution becomes unstable. On the other hand, for the nonvanishing intensity solutions (3.5) we get from (3.8)

$$
\lambda_{1,2}=\frac{1}{2}\left[\mu-1 \pm \sqrt{(\mu-1)^{2}-16 \mu}\right] .
$$

For $0<\mu<1$ the two eigenvalues have a negative real part, such that the nonzero intensity solutions are locally stable. As these two stable solutions coexist simultaneously, the global behavior is bistable. This result implies that the system presents a supercritical pitchfork bifurcation for $\mu=0 .{ }^{6}$ The limiting situation $\mu=1$, not considered in the previous analysis, can be discussed by noticing that, in this case of vanishing damping, the deterministic part of the system (3.1) becomes Hamiltonian,

$$
\begin{aligned}
& \dot{x}=x+v y\left(y^{2}+x^{2}\right)=\frac{\partial H(x, y)}{\partial y}, \\
& \dot{y}=-y-v x\left(y^{2}+x^{2}\right)=-\frac{\partial H(x, y)}{\partial x},
\end{aligned}
$$

with

$$
H(x, y)=x y+\frac{v}{4}\left(x^{2}+y^{2}\right)^{2} .
$$

In absence of fluctuations the system describes closed orbits of constant energy in the phase space as shown in Fig. 1. For initial conditions that correspond to negative values for the energy the system orbits around one of the steady states whereas for positive energies the orbits enclose the two steady states.

\section{FLUCTUATIONS AROUND STEADY STATES}

Small fluctuations $\delta x, \delta y$ around the steady states can be calculated from the linearized version of the stochastic equations (3.1),

$$
\left[\begin{array}{c}
\delta \dot{x} \\
\delta \dot{y}
\end{array}\right]=A\left[\begin{array}{l}
\delta x \\
\delta y
\end{array}\right]+\left[\begin{array}{c}
\widetilde{L}_{x} \\
\widetilde{L}_{y}
\end{array}\right],
$$

where the drift matrix $A$ is written out in Eq. (3.7). The stationary covariance matrix $\sigma_{i j} \equiv\left\langle x_{i} x_{j}\right\rangle-\left\langle x_{i}\right\rangle\left\langle x_{j}\right\rangle$ can be calculated in the linear approximation solving the matrix equation ${ }^{7}$

$$
A \sigma+\sigma A^{T}=-\bar{D} \text {. }
$$

\section{A. Below threshold}

According to (3.7), below threshold, the stationary mean values of $x$ and $y$ vanish and the covariance matrix becomes diagonal:

$$
\begin{aligned}
& \sigma_{11}=\left\langle x^{2}\right\rangle=\frac{\bar{d}}{2 \mu}=\frac{\gamma(1+2 \bar{n})}{4(\gamma-\kappa)}, \\
& \sigma_{22}=\left\langle y^{2}\right\rangle=\frac{\bar{d}}{2}=\frac{\gamma(1+2 \bar{n})}{4(\gamma+\kappa)} .
\end{aligned}
$$

It should be noted that in this case the linearization has completely eliminated the effect of the fourth-order term on the fluctuations, and the previous result is independent of $\Gamma$.

The zero-point fluctuations are $\left\langle x^{2}\right\rangle=\left\langle y^{2}\right\rangle=\frac{1}{4}$. The fluctuations in the $y$ component can be smaller than the zero-point ones provided that the gain verifies $\kappa>2 \gamma \bar{n}$. In that case the $y$ component is squeezed. Since the fluctuations are about the steady-state value $\left(x_{s}, y_{s}\right)=(0,0)$, this is a squeezed vacuum state. Note that the product of the mean squares is

$$
\left\langle x^{2}\right\rangle\left\langle y^{2}\right\rangle=\frac{1}{16} \frac{\gamma^{2}}{\gamma^{2}-\kappa^{2}}(1+2 \bar{n})^{2},
$$

that is greater than $\frac{1}{16}$. Thus, the state is not a minimum

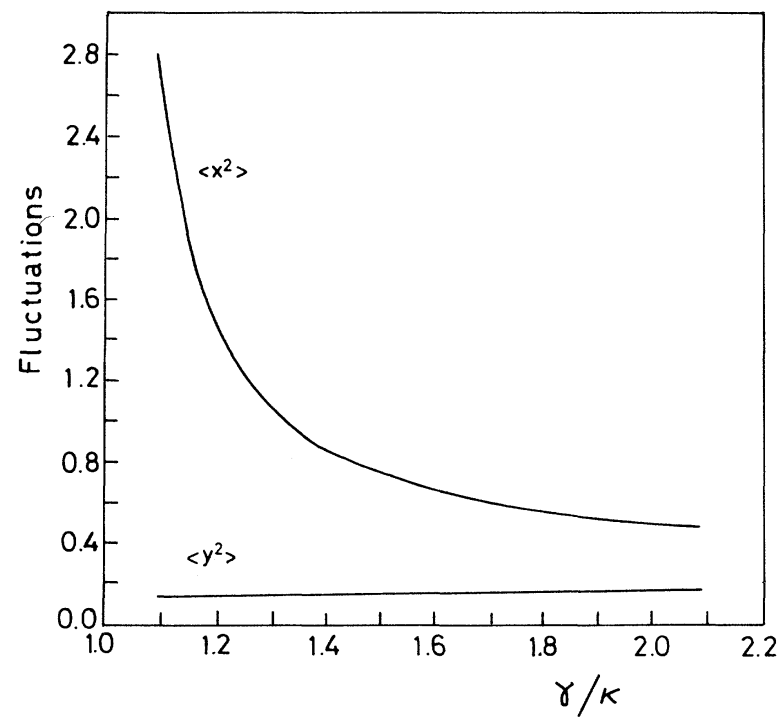

FIG. 2. Dependence of the fluctuations below threshold on the damping constant for $\bar{n}=0, \kappa=1$. 
uncertainty state.

Figure 2 shows the dependence of the fluctuations on the ratio between the damping constant $\gamma$ and the gain $\kappa$ at zero temperature $\bar{n}=0$. One can see that as we approach the threshold $\gamma / \kappa \rightarrow 1$, the squeezing in $y$ increases whereas the fluctuations for $x$ diverge. Near threshold the linearization around the stationary value is no longer valid. As a first approximation, nonlinear effects in the fluctuations can be taken into account by a Gaussian decoupling which consists in making in (3.1) the substitutions

$$
x^{2} \rightarrow\left\langle x^{2}\right\rangle, y^{2} \rightarrow\left\langle y^{2}\right\rangle,
$$

where $\left\langle x^{2}\right\rangle$ and $\left\langle y^{2}\right\rangle$ are the fluctuations in the linear approximation as given by Eq. (4.2). The resulting expression has the same formal structure as Eq. (4.1) with a drift matrix given by

$$
A=\left[\begin{array}{cc}
\mu & \frac{\bar{d} v}{2 \mu}(\mu-1) \\
-\frac{\bar{d} v}{2 \mu}(\mu-1) & -1
\end{array}\right] .
$$

Using (4.2) it is possible to calculate the covariance matrix. It turns out that the fluctuations in the $x$ and $y$ components become in this approximation

$$
\begin{aligned}
& \left\langle x^{2}\right\rangle=\frac{D}{2 \gamma} \frac{\gamma^{2}+\gamma \kappa+\left[\Gamma D \gamma /\left(\gamma^{2}-\kappa^{2}\right)\right]^{2}}{\gamma^{2}-\kappa^{2}+\left[\Gamma D \gamma /\left(\gamma^{2}-\kappa^{2}\right)\right]^{2}}, \\
& \left\langle y^{2}\right\rangle=\frac{D}{2 \gamma} \frac{\gamma^{2}-\gamma \kappa+\left[\Gamma D \gamma /\left(\gamma^{2}-\kappa^{2}\right)\right]^{2}}{\gamma^{2}-\kappa^{2}+\left[\Gamma D \gamma /\left(\gamma^{2}-\kappa^{2}\right)\right]^{2}} .
\end{aligned}
$$

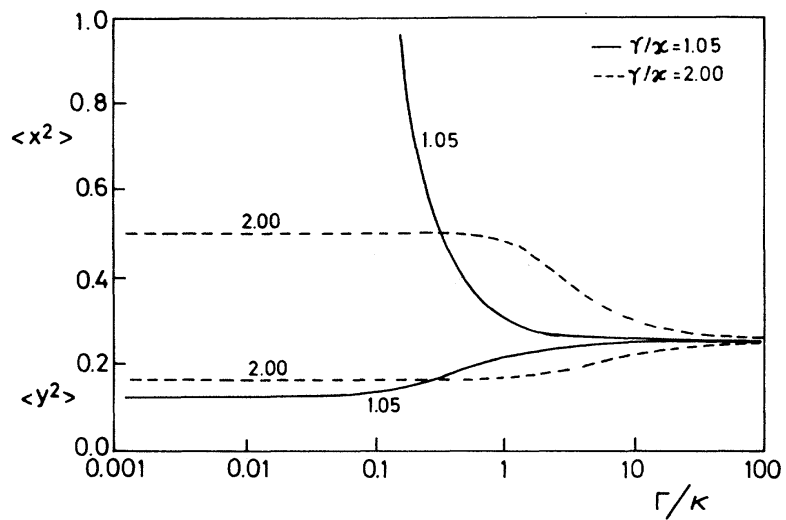

FIG. 3. Dependence of the fluctuations below threshold on the fourth-order coupling for $\gamma / \kappa=1.05$ ( - ) and 2.0 $(---)$.

Near threshold the nonlinearities become important. In fact for $\kappa \rightarrow \gamma$ we have $\left\langle y^{2}\right\rangle \rightarrow D /(2 \gamma)=(1+2 \bar{n}) / 4$, so the squeezing in the $y$ component vanishes even for $T=0$. For physical values of $\Gamma$ the effect of the nonlinear terms becomes important only close to the threshold as shown in Fig. 3 for zero temperature. However, now the correlation matrix $\sigma$ is not diagonal. The appropriate linear combination of $x$ and $y$ (that is, the appropriate phase of the electric field) which gives the minimum fluctuations can be obtained diagonalizing $\sigma$. In fact, the eigenvalues of $\sigma$ are the values of the minimum and maximum fluctuations. In this case we have

$$
\lambda_{1,2}=\frac{D}{2} \frac{\gamma\left\{1+\left[\Gamma D /\left(\gamma^{2}-\kappa^{2}\right)\right]^{2}\right\} \mp \kappa \sqrt{1+\left[\Gamma D /\left(\gamma^{2}-\kappa^{2}\right)\right]^{2}}}{\gamma^{2}-\kappa^{2}+\left[\Gamma D \gamma /\left(\gamma^{2}-\kappa^{2}\right)\right]^{2}}
$$

It can be shown that the minimum fluctuations $\lambda_{1}$ are always larger than the fluctuations for $\left\langle y^{2}\right\rangle$ given by (4.3), so that the effect of the nonlinear terms is to reduce the squeezing. According to the linear theory squeezing is maximized at threshold. However, the nonlinearities, whose effect is to decrease the squeezing, become also important close to threshold. There exists then a competition between these two effects so that for a fixed value of $\Gamma$ there is an optimum operating point to get the largest squeezing. For physical values of $\Gamma, \Gamma \sim 10^{-2}, 10^{-3}$, this point is very close to, but not at, the threshold.

In order to analyze the system of equations given in (2.7), a set of computer simulations of the classical trajectories have been carried out. ${ }^{8}$

The trajectories move around the trivial stationary solution. An example of these trajectories is shown in Fig. 4 for $\gamma / \kappa=1.02, \Gamma / \kappa=2 \times 10^{-2}$, and $\bar{n}=0$. It can be seen that the stationary probability distribution shows a strong asymmetry on both directions, this being a sig-

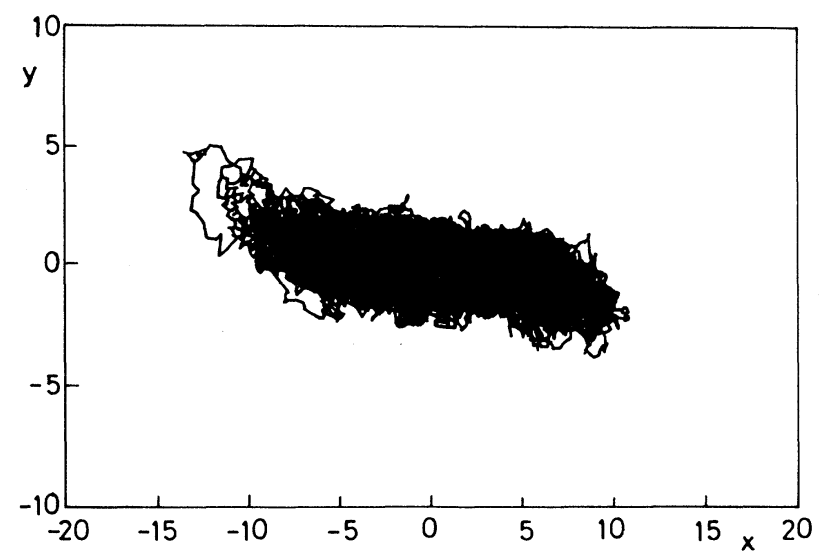

FIG. 4. Trajectory numerically determined from Eq. (2.7) for $\gamma / \kappa=1.02, \Gamma / \kappa=2 \times 10^{-2}$, and $\bar{n}=0$. 
nature of squeezing. It has also been seen that as $\gamma$ increases (i.e., $\gamma / \kappa=1.1$ ), the spherical symmetry is restored.

\section{B. Above threshold}

For $\mu>0$, linearizing around the nontrivial stationary solutions (3.5) leads to Eq. (4.1) but with a drift matrix:

$$
A=\left[\begin{array}{cc}
\mu \frac{\mu-1}{\mu+1} & \sqrt{\mu} \frac{3 \mu+1}{\mu+1} \\
-\sqrt{\mu} \frac{\mu+3}{\mu+1} & \frac{\mu-1}{\mu+1}
\end{array}\right]
$$

Solving Eq. (4.2) we find that the eigenvalues of the resulting covariance matrix are

$$
\begin{aligned}
& \sigma_{11}=\frac{\bar{d}}{16(1-\mu) \mu(1+\mu)^{2}}\left[B-\left(\mu^{2}-1\right) B^{1 / 2}\right], \\
& \sigma_{22}=\frac{\bar{d}}{16(1-\mu) \mu(1+\mu)^{2}}\left[B-\left(\mu^{2}-1\right) B^{1 / 2}\right], \\
& B=\mu^{4}+16 \mu^{3}+30 \mu^{2}+16 \mu+1 .
\end{aligned}
$$

Again, the linearization process gives fluctuations which are independent of the fourth-order coupling, $\Gamma$. Figure 5 plots $\sigma_{11}$ and $\sigma_{22}$ versus $\gamma / \kappa$. As $\gamma \rightarrow \kappa$ the fluctuations given by $\sigma_{11}$ become unbounded and the ones given by $\sigma_{22}$ tend to $\bar{d} / 2$. Note that since $\left(x_{s}, y_{s}\right)$ is real and not zero, the state may be described as phase-squeezed coherent.

According to (4.11) the maximum squeezing is obtained at threshold. But as in the case below threshold, close enough to it the linearization around the steady

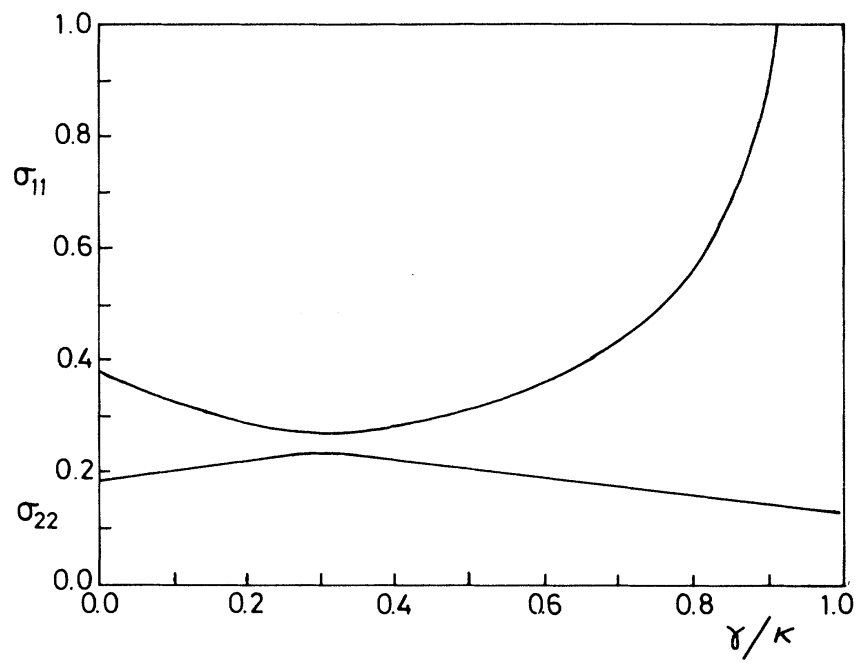

FIG. 5. Dependence of the fluctuations above threshold on the damping constant for $\bar{n}=0, \kappa=1$.

state is no longer valid. In this case the situation is even worse due to the fact that in this region the two nontrivial stationary states (3.5) are close enough for the existence of a noticeable probability of transition between the two states. This fact will be extensively discussed at the end of this section.

Introducing now a Gaussian correction, we found an effective drift matrix:

$$
A=\left[\begin{array}{cc}
\mu \frac{\mu-1}{\mu+1} & \sqrt{\mu \frac{1+3 \mu}{1+\mu}+\left(\sigma_{11}+\sigma_{22}\right) v} \\
-\sqrt{\mu} \frac{\mu+3}{\mu+1}+\left(\sigma_{11}+\sigma_{22}\right) v & \frac{\mu-1}{\mu+1}
\end{array}\right]
$$

As in the previous case the correlation matrix can be calculated using (4.2). In Fig. 6 is shown the dependence of the minimum and maximum fluctuations above threshold on the fourth-order coupling for various values of the damping constant and $\bar{n}=0$. It can be seen that the corrections introduced by the Gaussian approximation in the stationary correlations far from the threshold are practically negligible. Near threshold this approximation leads to a reduction of the squeezing. However, it should be noted that this Gaussian approximation does not take into account the jumps between the stationary states, which are more important near threshold, so it will not provide accurate results.

Numerical simulation allows us to compute the time interval, which depends on $\gamma$, on which the system jumps from one stable state to the other one. In Fig. 7 we show an example of a trajectory for $\gamma / \kappa=0.9, \Gamma / \kappa=2 \times 10^{-2}$, and $\bar{n}=0$. For this value of $\gamma / \kappa$, it can be seen that the two stationary stable states are appreciably separated.

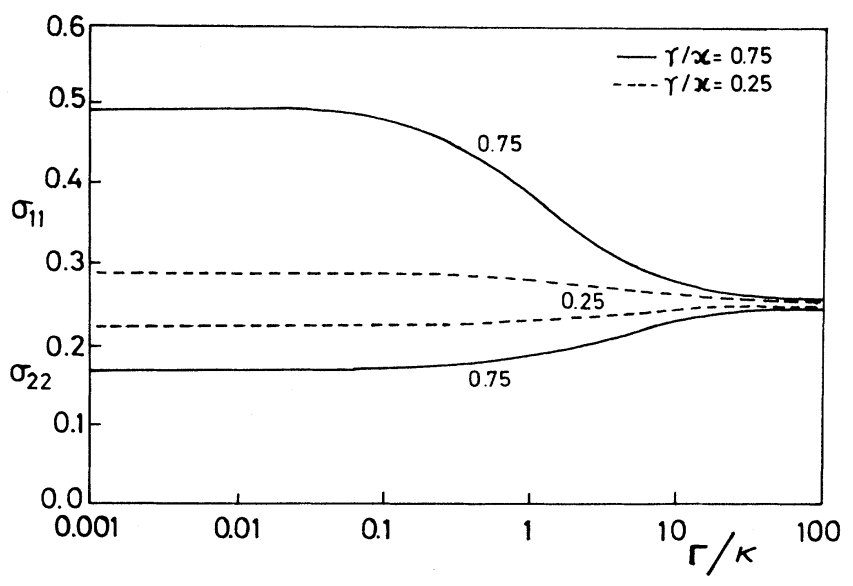

FIG. 6. Dependence of the fluctutations above threshold with Gaussian corrections on the fourth-order coupling for $\bar{n}=0$. The values of $\gamma / \kappa$ are $0.75(-), 0.25(---)$. 


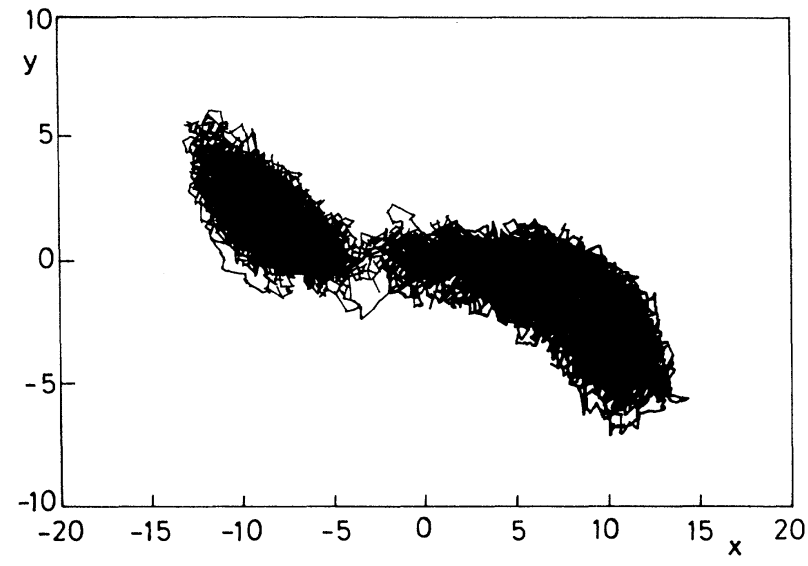

FIG. 7. Trajectory numerically determined from Eq. (2.7) for $\gamma / \kappa=0.9, \Gamma / \kappa=2 \times 10^{-2}$, and $\bar{n}=0$.

The probability of jumping between the two stable states decreases strongly with $\gamma$, in fact, for $\gamma / \kappa \sim 0.6$, jumps do not occur in observable times. A stationary probability distribution peaked around the two stationary stable states can be observed. As $\gamma$ approaches threshold (i.e., $\gamma / \kappa=0.98$ ), the two minima get closer and the jumps between them are more frequent, giving rise to a very broad structureless probability distribution function.

We have also considered the role of the strength of the nonlinearity. Decreasing the fourth-order coupling (i.e., $\left.\Gamma / \kappa=2 \times 10^{-3}\right)$, in the case above threshold, the two minima are further separated and the jumps between the stable states occur less frequently. In fact, for $\gamma / \kappa=0.9$, jumps do not occur in observable times for $\Gamma / \kappa=2 \times 10^{-3}$.

In Fig. 8 we compare the variances $\sigma_{11}$ and $\sigma_{22}$ numerically determined with the variances obtained from the linearized theory. It can be observed that for $\Gamma / \kappa=2 \times 10^{-2}$ (represented by circles in the figure), and above threshold, the value of $\sigma_{22}$ increases near threshold. This is due to the vicinity of the two minima for this value of $\Gamma$. If $\Gamma$ decreases (i.e., $\Gamma / \kappa=2 \times 10^{-3}$, represented by squares in the figure), the jumps between minima are very rare and the agreement with the linearized theory (continuous line) is excellent. This analysis makes clear the limits of validity of the linear analysis in the bistability domain.

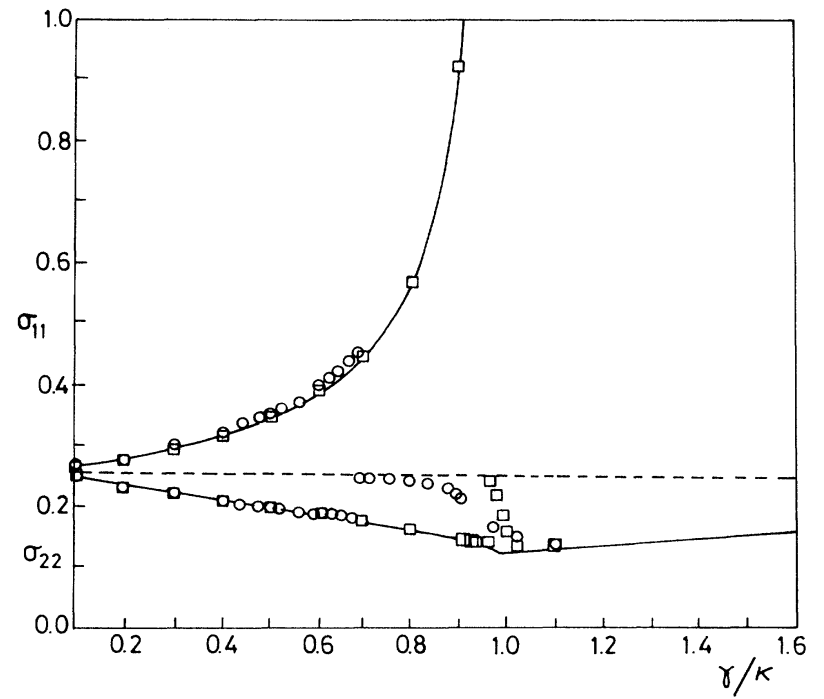

FIG. 8. Comparison between the variances numerically determined ( $O$ corresponds to $\Gamma / \kappa=2 \times 10^{-2}, \square$ to $\left.\Gamma / \kappa=2 \times 10^{-3}\right)$ and the variances determined from the linearized theory (solid line).

\section{CONCLUSIONS}

The role played by a fourth-order nonlinearity in a degenerate parametric amplifier has been studied in the present paper. As a result, it has become clear that the nonlinear contribution may perhaps be profitably used for the construction of above-threshold (bright) squeezing devices. In such a respect it is worth remarking that up to very recent times, ${ }^{9}$ the production of squeezed fields different from that of squeezed vacuum has presented a large number of experimental difficulties. Although some other squeezing mechanisms for the generation of intense squeezed light have also been proposed ${ }^{10}$ based on nonparametric devices, their experimental implementation seems to pose a large number of problems. On the other hand, the present results may indicate a way to control the adverse effects due to mode-hopping encountered in the operation above threshold of current parametric oscillators, ${ }^{11}$ since as it has been shown, the hopping rate can be controlled by a suitable choosing of the strength of the nonlinearity. Finally, the possibility of generating "oversqueezing" due to transient effects as suggested by Tombesi ${ }^{2}$ is being considered at the present moment and will be reported in due time.
${ }^{1}$ R. Tanas and S. Kielich, Quantum Opt. 2, 23 (1990).

${ }^{2} \mathrm{P}$. Tombesi, in Quantum Optics $I V$, edited by J. D. Harvey and D. F. Walls (Springer, Berlin, 1986), p. 81.

${ }^{3} \mathrm{~J}$. Perina, Quantum Statistics of Linear and Nonlinear Optical Phenomena (Kluwer Academic, Norwell, MA, 1984), Chap 7. ${ }^{4}$ C. C. Gerry, Phys. Lett. A 124, 237 (1987).

${ }^{5}$ T. W. Marshall and E. Santos, Phys. Rev. A 41, 1582 (1990); P. Kinsler and P. D. Drummond, Phys Rev. Lett. 64, 236 (1990); P. D. Drummond and P. Kinsler, Phys. Rev. A 40, 4813
(1989); H. J. Carmichael and M. Wolinsky, Phys. Rev. Lett. 60, 1836 (1988).

${ }^{6} \mathbf{J}$. Guckenheimer and P. Holmes, Nonlinear Oscillations, Dynamical Systems, and Bifurcations of Vector Fields (Springer-Verlag, New York, 1986).

${ }^{7}$ C. W. Gardiner, Handbook of Stochastic Methods (Springer, Berlin, 1985), Chap 4.

${ }^{8}$ Simulations are performed following the method indicated by J. M. Sancho, M. San Miguel, S. L. Katz, and J. D. Gunton, 
Phys. Rev. A 26, 1589 (1982). In all simulations we consider $\Gamma=0.01,0.001$. The time step of integration is $h=0.01$.

9 J. Mertz, A. Heidman, C. Fabre, E. Giacobino, and S. Reynaud, Phys. Rev. Lett. 64, 2897 (1990).

${ }^{10}$ F. A. M. de Oliveira and P. L. Knigth, in Dynamics of Non-
Linear Optical Systems, edited by L. Pesquera and F. J. Bermejo (World-Scientific, Singapore, 1986), p. 46.

${ }^{11}$ L. A. Wu, M. Xiao, and H. J. Kimble, J. Opt. Soc. Am. B 4, 1465 (1987). 